\title{
REVISION OF THE FAMILY SPHECIDAE (HYMENOPTERA, APOIDEA) IN IRAQ
}

\author{
Razzaq Shalan Augul \\ Department of Entomology and Invertebrates, Iraq Natural History Research \\ Center and Museum, University of Baghdad, Baghdad, Iraq \\ dr.rsha@nhm.uobaghdad.edu.iq, razzaqshalan@gmail.com
}

Received Date: 27 September 2019, Accepted Date: 04 December 2019, Published Date: 26 December 2019

\section{ABSTRACT}

A revision study of the Sphecidae from Iraq is presented. A survey is conducted to collect the specimens from different regions; generally, there were 41 species belonging to 12 genera and 4 subfamilies are revised with synonyms.

The current investigation included the species previously reported in Iraq, which were not collected during the current investigations; the distribution and other information are also provided.

Keywords: Hymenoptera, Iraq, Sphecidae, Revision, Wasps.

\section{INTRODUCTION}

The members of Sphecidae are cosmopolitan wasps that include: mud daubers, sand wasps, and other thread-waisted wasps; which can be distinguished by the following morphological characters: having a distinct petiole that composed of first abdominal sternite, with exception the species of Ammophila Kirby, 1798; it consist of two part first abdominal sternite and tergite; the inner orbit of compound eyes without notch; fore wings with three submarginal cells (exception some species in Ammophila and Prionyx Vander Linden, 1827; mandibles without notch; pronotal lobe rounded and separated by distinct distance from tegula; anal area of hind wing with wide jugal lobe and mesoscutum without notauli (Bohart and Menke,1976).

According to Pulawski (2019), the superfamily Apoidea consists from five families: Apidae, Heterogynaidae, Ampulicidae, Crabronidae and Sphecidae; globally, the last family consists of 785 species belonging to 19 genera, 4 subfamilies, these subfamilies including: Ammophilinae, Chloriontinae, Sceliphrinae and Sphecinae.

In Iraq; with the exception of some checklists that indicate the presence of many species within the Iraqi fauna (Beaumont, 1961; Khalaf, 1963; Derwesh, 1965; Kaddou, 1967) this family was studied in details at the first time by Augul (2012), but he considered it as a subfamily that contains three tribes: Ammophilini, Sphecini and Sceliphronini depending on Bohart and Menke (1976) in his study. Subsequently, several studies within this family including: Augul (2013), Augul et al. (2013, 2014, 2015).

The aim of this study is to establish a database for the species of the family Sphecidae in Iraq, with the treatment of the synonyms that contribute to processing the misidentification of 


\section{Revision of the family Sphecidae}

some species which preserved in the Iraq Natural History Research Center and Museum, with the possibility to add new information about this guild.

\section{MATERIALS AND METHODS}

The specimens were collected by aerial nets from different localities of Iraq for the period from February to end of November 2019; also used the undiagnosed specimens that previously collected and stored in the collection of Entomology and Invertebrates Department; Iraq Natural History Research Center and Museum (INHM).

The specimens were diagnosis by the author, and used many keys for this purpose, for example: Kohl (1918), Bohart and Menke (1976), Guichard (1986, 1988), Roche and Gadallah (1999), Roche (2007), Dollfuss (2013), Augul et al. (2013, 2014, 2015). General distribution base on Roche (2007), Gadallah et al. (2013) and Pulawski (2019).The information about synonyms was depended on: Honoré (1944), Dollfuss (2013, 2015 a, b), Pulawski (2019).

\section{Subfamily, Ammophilinae}

\section{RESULTS AND DISCUSSION}

Ammophila Kirby, 1798

Ammophila barbara (Lepeletier, 1845)

Synonym: Coloptera barbara Lepeletier de Saint-Fargean, 1845

Remark: this species very rarely in Iraq, there were two male specimens collected previously from Al-Hartha district, Basrah province (5.iv.1986) and deposited in INHM; the subsequent investigations revealed this species absent in Iraq.

Distribution: Africa: North Africa; Europe: Turkey; Asia: India, Israel, Jordan, Oman, Palestine, Syria, and UAE. Iraq (Augul et al., 2013).

Ammophila duhokensis Augul, Abdoul-Rassoul \& Kaddou, 2013

Materials (5 specimens): Dohuk province, Gara Mountain, (4 우, 1ठ) 16.vi.2019.

Distribution: Iraq (Augul et al., 2013).

Ammophila gracillima Taschenberg, 1869

Synonyms: Ammophila longicollis Kohl, 1884

Ammophila debilis F. Morawitz, 1889

Ammophila philomela Nurse, 1903

Materials (3 specimens): Wasit province, Aziziyah, Al Zelja village, (1 $\hat{\jmath}, 2$ 우) 6.v.2019.

Distribution: Africa: Algeria, Egypt, Ethiopia, Libya, Mauritania, Morocco, Niger, and Sudan. Europe: Turkey, Kazakhstan, and Russia. Asia: Afghanistan, China, Jordan, India, Iran, Israel, Oman, Saudi Arabia, Syria, Tajikistan, UAE, and Uzbekistan; Iraq (Augul et al., 2013).

Ammophila haimatosoma Kohl, 1884

Synonym: Sphex haimatosoma Turner, 1918

Distribution: Africa: Algeria, Chad, Cyprus, Egypt, Libya, Morocco, and Tunisia. Asia: Iran, Israel, Jordan, Saudi Arabia, UAE, and Yemen. Iraq (Kaddou, 1967). 
Razzaq Shalan Augul

Ammophila heydeni Dahlbom, 1845

Synonyms: Ammophila iberica Ed. André, 1886

Ammophila rubra Radoszkovski, 1876

Ammophila rubriventris A. Costa, 1864

Materials (11 specimens): Wasit, Al-Zubaidiyah district, Sher'han village, $(3 \hat{\jmath} \widehat{\jmath}, 8$ 우우) 11.viii.2019.

Distribution: Mediterranean Region, Part of central Europe; Central Asia, India,

Pakistan. In Iraq this species is registered by Kaddou (1967) under the name Ammophila heydeni velora Rad.

Ammophila hungarica Mocsary, 1883

Synonyms: Ammophila turcica Mocsáry, 1883

Ammophila hispanica Mocsáry, 1883

Ammophila fallax Kohl, 1884

Materials (7 specimens): Dohuk province, Amadi district, Deralok, ( 7 우) 17.vi.2019. Distribution: Africa: Algeria and Morocco. Europe: Armenia, Austria, Belgium, Bulgaria, Croatia, Cyprus, Czech Republic, France, Greece, Hungary, Italy, Kazakhstan, Poland, Portugal, Romania, Russia, Slovakia, Slovenia, Spain, Switzerland, Turkey. Asia: Afghanistan, India, Iran, Jordan, Syria, Tajikistan, Turkmenistan, and Uzbekistan; Iraq (Augul et al., 2013).

Ammophila occipitalis F. Morawitz, 1890

Synonym: Ammophila ruficollis F. Morawitz, 1890

Distribution: Europe: Russia, Turkey, Kazakhstan, Iran, Turkmenistan, and Mongolia. Iraq (Derwesh, 1965).

Remark: There are not specimens deposited in INHM; also during the current investigation, which conducted by the current author to survey about this family in different regions, proved that this species absent in Iraq from February 2010 till October 2019.

In Iraq, this species was reported by Derwesh (1965) under the synonym names Eremochares (Ammophila) occipitalis Morawitz and Sphex occipitalis.

Ammophila sabulosa (Linnaeus, 1758)

Synonyms: Ammophila cyanescens Dahlbom, 1845

Ammophila pulvillata Sowerby, 1806

Ammophila sabulosa Vander Linden, 1827

Ammophila vulgaris W. Kirby, 1798

Ichneumon frischii Geoffroy in Fourcroy, 1785

Podalonia sabulosa Bischoff, 1931

Psammophila sabulosa Zimmermann, 1935

Sphex dimidiatus Christ, 1791

Sphex hortensis Poda von Neuhaus, 1761

Sphex mucronatus Jurine, 1807 


\section{Revision of the family Sphecidae}

Distribution: Africa: Algeria.Europe: Albania, Austria, Belarus, Belgium, Bulgaria, Croatia, Czech Republic, Denmark, Finland, France, Germany, Great Britain, Greece, Hungary, Italy, Kazakhstan, Latvia, Lithuania, Luxemburg, Moldova, Netherlands, Norway, Poland, Portugal, Romania, Russia, Serbia, Slovakia, Slovenia, Spain, Sweden, Switzerland, Turkey, Ukraine. Asia: China, Iran, Japan, Korea, Kyrgyzstan, Mongolia, Nepal, Tajikistan, and Uzbekistan; Iraq (Augul et al., 2013).

Eremochares Gribodo, 1882

Eremochares dives (Brullé, 1833)

Synonyms: Ammophila elegans F. Smith, 1856

Ammophila festiva F. Smith, 1856

Eremochares doriae Gribodo, 1882:

Parapsammophila dives André, 1886

Parapsammophila retowskii Konow, 1887

Sphex dives Turner, 1917

Materials (2 specimens): Wasit, Al -Zubaidiyah district, Sher'han village, (2 $\circ$ ) 1.vi.2019.

Distribution: Africa: Algeria, Egypt, Libya, Morocco, and Tunisia. Europe: Greece, Kazakhstan, Russia, Turkey, and Turkmenistan. Asia: Afghanistan, Bahrain, China, India, Iran, Israel, Lebanon, Oman, Saudi Arabia, Syria, and Uzbekistan. Iraq (Morice, 1921).

Remark: This species registered in Iraq by Morice (1921) under the synonym Sphex dives, whereas Derwesh (1965) registered it under the synonym Sphex (Eremochares) dives Turner, 1917.

Parapsammophila Taschenberg, 1869

Parapsammophila turanica F. Morawitz, 1890

Synonyms: Ammophila turanica Dalla Torre, 1897

Eremochares lutea Myartseva, 1971

Distribution: Africa: Algeria, Egypt, Morocco, and Libya. Europe: Kazakhstan. Asia: Israel, Jordan, Kuwait, Oman, Saudi Arabia, Turkmenistan, and UAE; in Iraq this species registered by Augul et al. (2013).

Podalonia Fernald, 1927

Podalonia ebenina (Spinola, 1839)

Synonyms: Ammophila hirsuta var. ebenina Zavattari, 1909

Ammophila mandibulata W.F. Kirby, 1889

Ammophila micipsa Kohl, 1905

Ammophila (Psammophila) micipsa Morice, 1900

Podalonia ebenina Leclercq, 1955

Psammophila ebenina A. Costa, 1864

Psammophila micipsa Morice, 1900

Distribution: Africa: Algeria, Egypt. Europe: Armenia, Caucasus, France, Greece, Italy, Kazakhstan, Russia, Turkey. Asia: Afghanistan, Iran, Israel, Jordan, Kuwait, Lebanon, Tajikistan, Turkmenistan, and Uzbekistan; Iraq (Beaumont, 1961). 
Razzaq Shalan Augul

Podalonia marismortui (Bytinski - Salz, 1955)

Distribution: Iraq (Beaumont, 1961); Asia: Israel, Egypt and Jordan.

Podalonia minax (Kohl, 1901)

Synonyms: Ammophila confalonierii Guiglia, 1932

Ammophila minax Kohl, 1901

Distribution: Africa: Algeria, Egypt, and Morocco. Asia: Iran, Kuwait, Saudi Arabia. Iraq (Augul et al., 2013).

Podalonia tydei (Le Guillou, 1841)

Synonyms: Ammophila capuccina A. Costa, 1858

Ammophila errabunda Mercet, 1906

Ammophila homogenea Mercet, 1906

Ammophila klugii Lepeletier de Saint Fargeau, 1845

Ammophila lanuginosa Marquet, 1881

Ammophila psammodes Lepeletier de Saint Fargeau, 1845

Ammophila tydei Le Guillou, 1841

Psammophila madeirae Dahlbom, 1843

Psammophila senilis Dahlbom, 1843

Psammophila tydei (Le Guillou, 1841)

Sphex tydei (Le Guillou, 1841)

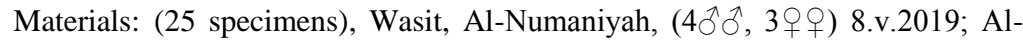

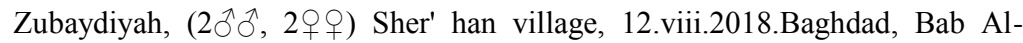

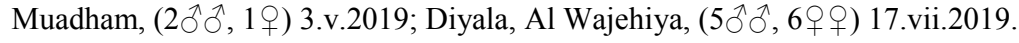
Distribution: Africa: Algeria, Cameron, Chad, Egypt, Eritrea, Kenya, Libya, Morocco, Niger, Tanzania, Rwanda, South Africa, Sudan, Tunisia, Uganda, and Zimbabwe. Europe: Austria, Bulgaria, Cyprus, Czech Republic, Finland, France, Greece, Germany, Hungary, Italy, Kazakhstan, Madeira, Malta, New Zealand, Poland, Portugal, Romania, Russia, Slovakia, Spain, Turkey, Ukraine, and Uzbekistan; Australia; Asia: Afghanistan, China, India, Iran, Israel, Jordan, Kuwait, Kyrgyzstan, Oman, Saudi Arabia, Syria, Tajikistan, Turkmenistan, UAE, and Yemen; Iraq (Beaumont, 1961).

\section{Subfamily, Chloriontinae}

Chlorion Latreille, 1802

Chlorion semenowi F. Morawitz, 1890

Synonym Sphex semenowi Kohl, 1890

Distribution: Iraq (Morice, 1921); Iran, Turkmenistan and Russia.

Remark: There are no specimens collected during the current investigation, and not found in the collection of INHM.

Chlorion funereum Gribodon, 1879

Synonyms: Sphex eximius Kohl, 1885

Sphex funereus Kohl, 1885

Sphex kohli Ed. André, 1888

Distribution: Iraq (Roche, 2007) South Africa: Algeria, Egypt, Somalia to Chad, Sudan Asia: Yemen and Saudi Arabia. 


\section{Subfamily, Sphecinae}

Chilosphex Menke, 1976

Chilosphex argyrius (Brullé, 1833)

Synonyms: Chlorion argyrius (Brulle, 1833)

Sphex argyrius Brullé, 1833

Sphex emarginatus Brullé, 1833

Distribution: Africa: Algeria; Europe: Croatia, Greece, Italy, Kazakhstan, Spain, France, Asia: Iran, Turkey, and Russia. Iraq (Morice, 1921).

Remark: This species registered by Morice (1921) in Iraq under the name Chlorion argyrius; did not get any specimens during the surveys of different localities of Iraq for the period from February 2010 to September 2019; also there are no specimens belonging to this species in the collection of INHM.

Isodontia (Patton, 1880)

Isodontia albohirta (Turner, 1908)

Synonym: Sphex albohirtus Turner (1908)

Distribution: Australia (Bohart and Menke, 1976).

Remark: Kaddou (1967) listed this species in Iraq under the name Sceliphron albohirtus Turner (1908).

Palmodes Kohl, 1890

Palmodes melanarius (Mocsáry, 1883)

Synonym: Chlorion melanarius Mocsary, 1883

Sphex melanarius Mocsáry, 1883

Sphex anatolicus Kohl, 1888

Sphex anatolicus Kohl, 1888

Sphex picicornis F. Morawitz, 1890

Distribution: Africa: Algeria, Libya and Morocco. Europe: Greece, Kazakhstan, Portugal, Russia, Spain, Turkey, Ukraine. Asia: Iran, Syria, Kyrgyzstan, Tajikistan, and Turkmenistan.

Remark: Morice (1921) listed this species in Iraq under the synonym name Chlorion melanarius Mocsary, 1883; whereas Beaumont (1961) listed it in Iraq under the valid name. We did not get any specimens during the surveys of different localities of Iraq for the period from February 2010 to September 2019; also there are no specimens belonging to this species in the collection of INHM.

Prionyx Vander Linden, 1827

Prionyx crudelis (F. Smith, 1856)

Synonyms: Harpactopus crudelis F. Smith, 1856

Pepsis hirtipes Fabricius, 1804

Sphex rufipennis Fabricius, 1793

Sphex aegyptius Lepeletier de Saint Fargeau, 1845

Sphex crudelis de Beaumont, 1949

Sphex grandis Radoszkowski, 1876

Sphex soror Honoré, 1944

Materials (4 specimens): Baghdad province, Bab Al Muadham, (1 $\widehat{\jmath}, 2$ 웅) 21.vii.2019; (1ㅇ) 7.viii. 2019.

Distribution: Africa: Algeria, Cameron, Egypt, Eritrea, Ethiopia, Guinea, Kenya, Madagascar, Mauritania, Somalia, Sudan, and Tanzania. Europe: Bulgaria, Cyprus, Greece, Kazakhstan, and Turkey. Asia: India, Iran, Kuwait, Saudi Arabia, Oman, Philippines, Tajikistan, Turkmenistan, UAE, and Yemen; Iraq (Beaumont, 1961). 


\section{Razzaq Shalan Augul}

Remark: In Iraq this species is registered by Beaumont (1961) under the name Sphex crudelis de Beaumont, 1949; then Derwesh (1965) listed this species under the name of Chlorion crudele.

Prionyx kirbii (Vander Linden, 1827)

Synonyms: Ammophila kirbii Vander Linden, 1827

Sphex albisectus Lepeletier de Saint Fargeau and Serville, 1828

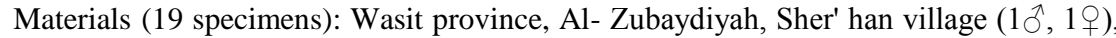

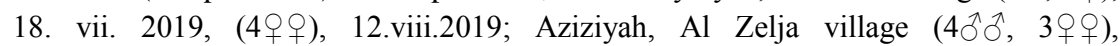
12.viii.2019. Baghdad, Bab Al Muadham, (1今, 3우), 5.ix.2019. Karbala province, (2웅) 18.ix.2019.

Distribution: Africa: Algeria, Cameron, Egypt, Eritrea, Ethiopia, South Africa, Tanzania, Tunisia, and Libya. Europe: Austria, Belarus, Bulgaria, Croatia, Czech Republic, France, Germany, Greece, Hungary, Italy, Portugal, Romania, Russia, Slovakia, Spain, Switzerland, Turkey, Kazakhstan, Malta, Portugal, and Ukraine. Asia: Uzbekistan, China, Iran, Tajikistan, Syria and Israel; in Iraq this species registered by Augul et al. (2015).

Prionyx kurdistanicus (Balthasar, 1954)

Synonym: Sphex kurdistanicus Balthasar, 1954

Distribution: Iraq (Balthasar, 1954).

Remark: There are no specimens belonging to this species in the collection of INHM; also did not collect any specimens during the various our investigations.

Prionyx lividocinctus (A. Costa, 1801)

Synonym: Enodia graeca Mocsáry, 1883

Enodia lividocincta A. Costa, 187

Enodia obliquestriata Mocsáry, 1883

Priononyx isselii Gribodo, 1880

Sphex micans Ed. André, 1888

Distribution: Africa: Egypt, Libya, Morocco, and Tunisia. Europe: Bulgaria, Cyprus, France, Greece, Italy, Kazakhstan, Spain, Portugal, Russia, and Turkey. Asia: Iran, Kyrgyzstan, Tajikistan, Turkmenistan, and Uzbekistan.

Remark: In Iraq this species is registered by Kaddou (1967) under the name Sphex lividocinctus Corte.

Prionyx macula (Fabricius, 1804)

Synonyms: Pepsis macula Fabricius, 1804

Sphex eatoni E. Saunders, 1910

Distribution: Africa: Algeria, Egypt, Kenya, and Libya. Europe: Kazakhstan; Asia: Afghanistan, Iran, Israel, Kuwait, and Saudi Arabia. Iraq (Beaumont, 1961).

Remark: The members of this species are very rare; there is one male specimen only that stored in the collection of the INHM.

Prionyx niveatus (Dufour, 1854)

Synonyms: Calosphex niveatus (Dufour, 1854)

Enodia albopictinata Taschenberg, 1869

Podium maracandicum Radoszkowski, 1877

Prionyx afghaniensis (de Beaumont, 1970)

Sphex afghaniensis de Beaumont, 1970

Sphex niveatus Dufour, 1854 
Revision of the family Sphecidae

Sphex maracandica (Radoszkowski, 1877)

Sphex suavis F. Morawitz, 1893

Distribution: Africa: Algeria, Egypt, Libya, Malawi, Mauritania, Morocco, Niger, South Africa, Sudan, Tunisia, and Mongolia. Europe: Turkey and Kazakhstan; Asia: Afghanistan, Iran, Israel, Jordan, Kuwait, Oman, Saudi Arabia, Turkmenistan, UAE, Uzbekistan, and Yemen. Iraq (Augul et al., 2015).

Prionyx songaricus (Eversmann, 1849)

Synonyms: Sphex songaricus Eversmann, 1849

Sphex tenuicornis F. Morawitz, 1890

Distribution: Africa: Algeria; Europe: Kazakhstan, Russia, and Turkey. Asia: Afghanistan, Iran, Israel, Syria, Tajikistan, Turkmenistan, and Uzbekistan; Iraq (Beaumont, 1961).

Remark: There is no information about this species in Iraq.

Prionyx stschurowskii (Radoszkowski, 1877)

Synonym: Sphex stschurowskii Radoszkowski, 1877

Distribution: Africa: Algeria, Egypt, Morocco, and Tunisia. Europe: Kazakhstan; Asia: Afghanistan, Iran, Saudi Arabia Turkmenistan, and Uzbekistan; Iraq (Beaumont, 1961). Remark: Derwesh (1965) listed this species in Iraq under the name Chlorion hyalipenne; the members of species are very rare in the field, there is one female only found in the collection of the INHM.

Prionyx subfuscatus (Dahlbom, 1845)

Synonyms: Enodia chrysoptera Ruthe and Stein, 1857

Gastrosphaeria anthracina A. Costa, 1858

Sphex desertorum Eversmann, 1849

Sphex nigritus Lucas, 1849

Sphex soror Dahlbom, 1845

Sphex subfuscatus Dahlbom, 1845

Distribution: Africa: Algeria, Egypt, Ethiopia, Libya, Mongolia, and Morocco. Europe: Albania, Croatia, Cyprus, Czech Republic, France, Germany, Greece, Hungary, Italy, Kazakhstan, Portugal, Romania, Russia, Slovakia, Spain, Turkey, Ukraine. Asia: China, India, Iran, Israel, Korea, Kyrgyzstan, Oman, Saudi Arabia, Tajikistan, Turkmenistan, Uzbekistan.

Remark: El-Haidari et al. (1971) listed this species in Iraq under the synonym Sphex subfasciatus Dahlbom, 1845; to addition, there is no information about this species in Iraq, especially in the INHM.

Sphex Linnaeus, 1758

Sphex flavipennis Fabricius, 1793

Synonyms: Ammophila flavipennis Valetta, 1979

Pepsis flavipennis Fabricius, 1804

Pelopaeus flavipennis Stephens, 1829

Sphex bicolor Dahlbom, 1845

Sphex cinereorufocinctus Dahlbom, 1845

Sphex sellae Gribodo, 1873

Materials (2 specimens): Dohuk, Gara Mountain, (2웅) 18.vi.2019.

Distribution: Africa: Algeria, Egypt, Libya, Morocco, and Tunisia. Europe: Bulgaria, Croatia, Cyprus, France, Greece, Hungary, Italy, Kazakhstan, Macedonia, Malta, 


\section{Razzaq Shalan Augul}

Portugal, Romania, Russia, Spain, Turkey, UK, and Ukraine. Asia: Afghanistan, China, Iran, Israel, Kyrgyzstan, Palestine, Syria, Tajikistan, Turkmenistan, and UAE, and Uzbekistan; Iraq (Morice, 1921).

Sphex funerarius Gaussakovskij, 1934

Synonyms: Sphex maxillosus Fabricius, 1793

Sphex obscurus Fischer de Waldheim, 1843

Sphex rufocinctus Brullé, 1833

Distribution: Iraq (Kaddou, 1967); Egypt, Algeria, Morocco, Tunisia, Libya, Macedonia Malta, Bulgaria, Germany, UK, Croatia, Italy, Austria, Ukraine, Hungary, Spain, Slovakia, Slovenia, former Yugoslavia, France, Portugal, Poland, Greece, Romania, Czech, Switzerland, Russia, Kyrgyzstan, Uzbekistan, Kazakhstan, Tajikistan, Turkmenistan, Turkey, Syria, China. Iraq (Kaddou, 1967).

Remark: Kaddou (1967) reported this species in Iraq under the name Sphex maxillosus Fabricius, 1793.

Sphex leuconotus Brullé, 1833

Synonyms: Sphex triangulum Brullé, 1833

Sphex sordidus Dahlbom, 1845

Sphex tristis Kohl, 1885

Sphex plumipes Radoszkowski, 1886

Sphex pachysoma Kohl, 1890

Distribution: Iraq (Beaumont, 1961); Algeria, Morocco, Greece, Bulgaria, Kazakhstan, Turkey, Italy, Iran, Azerbaijan, Jordan, Kazakhstan, Kyrgyzstan, Syria, Tajikistan, Turkmenistan, and Uzbekistan, Spain, Ukraine, Afghanistan, Romania, Russia, France, Israel and Cyprus.

Remark: didn't get specimens of this species during the current investigation, also there are no specimens preserved in the collection of Iraq Natural History Research Center and Museum.

Sphex pruinosus Germar, 1817

Synonyms: Sphex vicinus Lepeletier de Saint Fargeau, 1845

Sphex scioensis Gribodo, 1879

Sphex rothneyi Cameron, 1889

Sphex retractus Nurse, 1903

Materials (19 specimens): Wasit province, Az Zubaydiyah, Sher' han village,

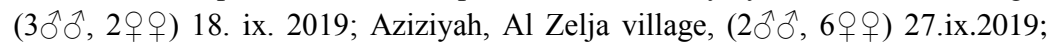

Sulaymaniyah province, Kunamasi, (2 $\hat{\jmath} \delta^{\lambda}, 4$ 우우) 21.viii.2019.

Distribution: Iraq (Kaddou, 1967); Algeria, Tunisia, Ethiopia, Morocco, Libya, Lebanon, Yemen, UAE, Oman, Saudi Arabia, Syria, Egypt, Iran, Israel, France, Albania, Malta, Greece, Bulgaria, Croatia, Cyprus, Spain, Italy, Macedonia, Hungary, Portugal, Russia, Afghanistan, Kazakhstan, Uzbekistan, Turkmenistan, India, Pakistan, Kyrgyzstan, Tajikistan and Turkey.

Sphex zubaidiyacus Augul, 2013

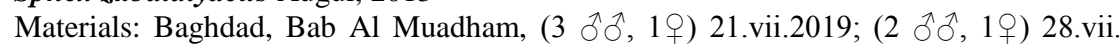
2019.

Distribution: Iraq (Augul, 2013).

Remark: In the present investigation, a new locality in Iraq was registered for this species. 


\section{Subfamily, Sceliphrinae}

Chalybion Dahlbom, 1843

Chalybion bengalense (Dahlbom, 1845)

Synonyms: Pelopoeus bengalense Dahlbom, 1845

Pelopoeus convexus F. Smith, 1876

Sphex violaceus Fabricius, 1775

Distribution: In Iraq, it was registered as Chalybion bengalense F. Smith, 1871 by Derwesh (1965); South Africa, Tanzania, Madagascar, Ethiopia, Yemen, Maldives, Eritrea, Mozambique, Bangladesh, Egypt, Greece, Nepal, Italy, Sri Lanka, Vietnam, India, Burma, Malaysia, Singapore, Thailand, China, Taiwan, Japan, Philippines, Indonesia, Timor, Australia and United State.

Chalybion flebile (Lepeletier de Saint Fargeau, 1845)

Synonyms: Pelopoeus flebilis Lepeletier de Saint Fargeau, 1845

Pelopoeus targionii Carruccio, 1872

Sceliphron targionii Dalla Torre, 1897

Materials (34 specimens): Wasit province, Al-Zubaydiyah,, Sher' han village, (4 $\widehat{\delta}^{\lambda}$,

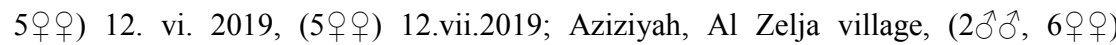

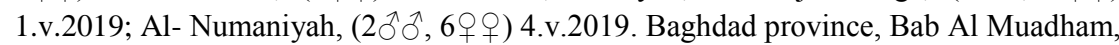
$(3 \hat{\jmath}, 1$ 우) 5.v.2019.

Distribution: Iraq ( Beaumont,1961); Turkey, Greece, Italy, Egypt, Saudi Arabia, Iran, France, UAE, Jordan, Morocco, Oman, Spain, Syria, Tunisia, Algeria, Libya, Cyprus, Kazakhstan and Portugal.

Sceliphron Klug, 1801

Sceliphron arabs (Lepeletier de Saint Fargeau, 1845)

Synonyms: Pelopaeus arabs Lepeletier de Saint Fargeau, 1845

Pelopaeus caucasicus Ed. André, 1888

Sceliphron caucasicum Dalla Torre, 1897

Materials (25 specimens): Wasit province, Al-Zubaydiyah,, Sher' han village, $\left(2 \delta^{\lambda}\right.$,

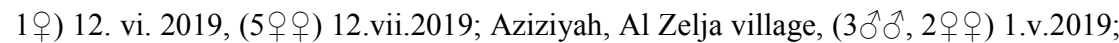
Al-Numaniyah, (5우) 4.v.2019. Sulaymaniyah province, Kunamasi, (1ภ, 1우) 21.viii. 2019; Dokan $(4 \widehat{\jmath}, 1$ 우) 24.viii. 2019.

Distribution: Iraq (Morice, 1921); Algeria, Iran, Turkey and Syria.

Remark: Morice (1921) listed this species under the synonym Sceliphron caucasicum.

Sceliphron curvatum (F. Smith, 1870)

Synonym: Pelopaeus curvatus F. Smith, 1870

Distribution: Iraq (Abdul Rassoul,1976); Turkey, Afghanistan, India, Tajikistan, Uzbekistan, Pakistan, Nepal, Kyrgyzstan, Montenegro, Belgium, Italy, Czech Republic, Croatia, Hungary, France, Switzerland, Spain, Slovakia, Austria, Kazakhstan, Ukraine, Russia, Germany, Serbia, Chile, Poland, Slovenia and Bulgaria.

Remark: This species listed by Abdul Rassoul (1976) under the name Sceliphron deforme, and then corrected to Sceliphron curvatum by Hensen (1987).

Sceliphron madraspatanum (Fabricius, 1781)

Synonyms: Pelopoeus interruptus Palisot de Beauvois, 1806

Pelopaeus bilineatus F. Smith, 1852

Pelopoeus separatus F. Smith, 1852

Sphex madraspatanus Fabricius, 1781 
Razzaq Shalan Augul

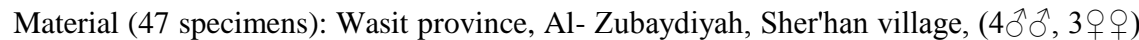

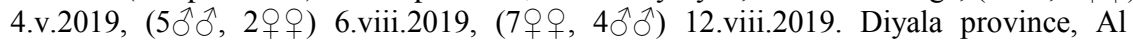

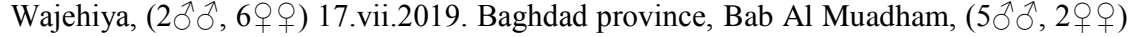
29.iv.2019. Dohuk, Sarsink, 18.vi.2019 (3웅). Sulaymaniyah province, Kunamasi, (4우우) 22.viii.2019.

Distribution: Iraq (Beaumont, 1961); Afghanistan, Bangladesh, China, India, Indonesia, Syria, Iran, Oman, UAE, Pakistan, Kyrgyzstan, Turkmenistan, Tajikistan, Uzbekistan, Kazakhstan, Japan, Malaysia, Nepal, Thailand, Taiwan, Philippines, Sri Lanka, Vietnam, Ukraine, Russia, Greece, Turkey, France, Madagascar, Bulgaria, Croatia, Italy, Laos and Montenegro.

Remark: Morice (1921) listed the subspecies Sphex madraspatanum tubifex Latreille, 1809 in Basrah province; whereas Schmid-Egger (2011) listed the subspecies, Sceliphron madraspatanum pictum (F. Smith, 1856)

Sceliphron pietschmanni Kohl, 1918

Distribution: Iraq (kohl, 1918); Syria and Iran.

Remark: This species is very scarce in Iraq.

Sceliphron rectum Kohl, 1918

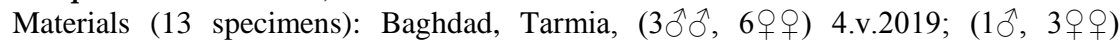
31.vi.2019.

Distribution: Iraq (Augul, 2014); Saudi Arabia, Iran and India.

Remark: We noticed that this species present only in Baghdad during field surveys from 2010 till that conducted in the previous and current studies.

Sceliphron spirifex (Linnaeus, 1758)

Synonyms: Sphex aegyptius Linnaeus, 1758

Sphex flavipes Christ, 1791

Sphex spirifex Linnaeus, 1758

Sphex spirifex atra Scopoli, 1786

Distribution: Iraq (Khalaf and Al-Omar, 1974); southern Europe, Africa, Asia; widespread in Arabia, including the United Arab Emirates.

\section{LITERATURE CITED}

Abdul-Rassoul, M. S. 1976. Checklist of Iraq natural history museum insects collection. Natural History Research Center, Publication, 30: 1-41.

Augul, R. S. 2012. Taxonomic study of Thread-Waisted Wasps Subfamily: Sphecinae (Hymenoptera: Sphecidae) from some Governorates of Iraq. Ph. D. thesis, Department of Biology, College of Science, University of Baghdad, 286 pp. (In Arabic).

Augul, R. S. 2013. A new species of the genus Sphex Linnaeus, 1850 (Hymenoptera: Sphecidae; Sphecinae) from Iraq. International Journal of Advanced Research, 1 (5): 475-484.

Augul, R. S., Abdul-Rassoul, M. S. and Kaddou, I. K. 2013. A new species of ammophila kirby, 1798 with identification key to species of Ammophilini (Hymenoptera: Sphecidae: Sphecinae) in Iraq. Advances in Bioresearch, 4 (1): 12-27. 
Augul, R. S., Abdul-Rassoul, M. S. and Kaddou, I. K. 2015. Identification key to species of sphecini (Hymenoptera: Sphecidae: Sphecinae) in Iraq. Journal of Biodiversity and Environmental Sciences, 6 (1): 111-121.

Augul, R. S., Abdul-Rassoul, M. S., Kaddou, I. K. and Jihad, H. M. 2014. Identification Key to Species of Sceliphrini (Hymenoptera: Sphecidae: Sphecinae) with illustration of male genitalia in Iraq. International Journal of Current Microbiology and Applied Sciences, 3 (4): 663-670.

Balthasar, V. 1954. Příspěvek k poznání palestinských kutilek - Ein Beitrag zur Kenntnis der palästinischen Spheciden. Opuscula hymenopterologica XIV. Acta Entomologica Musei Nationalis Pragae, 28:267-284.

Beaumont, J.de.1961. Sphecidae de l'Iraq (Hym.). Opuscula Zoologica (München), 56:1-5. Available http://researcharchive.calacademy.org/research/entomology/Entomology_Resources /Hymenoptera/sphecidae/copies/de_Beaumont_1961e_Iraq.pdf

Bohart, R. M. and Menke, A. S. 1976. Sphecid wasps of the world: A generic revision. University of California Press, Berkeley, California, 695pp.

Derwesh, A. I. 1965. A preliminary list of identified insects and arachnids of Iraq. Director General Agriculture Research Projections Baghdad, Bulletin, no. 121, 123pp.

Dollfuss, H. 2013. Revision of the wasps genus Ammophila Kirby 1798 (Hymenoptera: Apoidea: Sphecidae) of the Palearctic Region and India. Linzer biologische Beiträge, 45(1): 383-564.

Dollfuss, H. 2015a. Revision of the wasp genus Ammophila KIRBY, 1798 (Hymenoptera: Apoidea: Sphecidae) of the Sub-Saharan Region of Africa. Linzer Biologische Beiträge, 47: 307-412.

Dollfuss, H. 2015b. The Ammophilini wasps of the "Biologiezentrum Linz"- collection in Linz, Austria (part 3) including the genera Ammophila Kirby, Eremnophila Menke, Eremochares Gribodo, Hoplammophila de Beaumont and Podalonia Fernald (Hymenoptera, Apoidea, Sphecidae). Linzer Biologische Beiträge, 47: 413-439.

El - Haidari, H., Fattah, Y. M. and Sultan, J. A. 1971. Contribution to the insect fauna of Iraq (part 3). Ministry Agricultural Iraq, Bulletin, no.3, 20pp.

Gadallah, N. S., AL Dhafer, H. M., Al Dryhim, Y. N., Fadl, H. H. and El Gharbawy, A. A. 2013. The digger wasps of Saudi Arabia: New records and distribution, with a checklist of species (Hym.: Ampulicidae, Crabronidae and Sphecidae). North Western Journal of Zoology, 9 (2):345-364. 


\section{Razzaq Shalan Augul}

Guichard, K. M. 1986. Hymenoptera: Fam. Sphecidae of Arabia. Key to the Arabian genera of hunting wasps. Fauna of Saudi Arabia, 8: 343-351.

Guichard, K. M. 1988. Hymenoptera: Sphecidae: Subfam. Sphecinae of the Arabian Peninsula. Fauna of Saudi Arabia, 9: 114-131.

Hensen, R. V.1987. Revision of the subgenus Prosceliphron van der Vecht (Hymenoptera, Sphecidae). Tijdschrift voor Entomologie, 129:217-261.

Honoré, A. M. 1944. Revue des espèces égyptiennes du genre Sphex Linné, 1758 (Hymenoptera: Sphegidae). Bulletin de la Société Fouad 1er Entomologique, 28: 45-79.

Kaddou, I. K. 1967. Checklist of some insects fauna of Iraq. Biological Research Centre, Publication, no. 1, 44pp.

Khalaf, K. T.1963. Faunistic notes in Iraq. Bulletin of the Iraq Natural History Museum, 11(8):8-9.

Khalaf, A. N. and Al - Omar, M. A. 1974. A second list of insects from Iraq. Biological Research Centre, Publication, no. 2, 41pp.

Kohl, F. F. 1918. Die Hautflüglergruppe "Sphecinae". IV. Die natürliche Gattung Sceliphron Klug (= Pelopaeus Latr.). Annalen des k.k. Naturhistorischen Hofmuseums, 32: 1171. Available at: http://biodiversitylibrary.org/page/5585249

Morice, F. D. 1921. Annotated lists of Aculeate Hymenoptera (except Heterogyna) and Chrysids recently collected in Mesopotamia and North-West Persia. The Journal of the Bombay Natural History Society, 27:816-828.

Pulawski, W. J. 2019. Catalog of Sphecidae. California Academy of Sciences, San Francisco. https://www.calacademy.org/scientists/projects/catalog-of-sphecidae (Accessed at July 2019)

Roche, C. G. 2007. Conspectus of the Sphecid wasps of Egypt (Hymenoptera: Ampulicidae, Sphecidae, Crabronidae). Egyptian Journal of Natural History, 4: 12 - 149. Crabronidae).

Roche, G. C. and Gadallah, N. S. 1999. The sphecid wasps of Egypt (Hymenoptera: Sphecidae): Introduction and generic key. Egyptian Journal of Biology, 1:104-117.

Schmid-Egger, C. 2011. Order Hymenoptera, families Crabronidae and Sphecidae. Arthropod fauna of the UAE, 4: 488-608. 


\title{
مراجعة لعائلة Sphecidae (غشائية الاجنحة، النحليات) في العراق
}

\author{
رزاق شعلان عكل \\ مركز بحوث و متحف التأريخ الطبيعي/ جامعة بغداد عذل

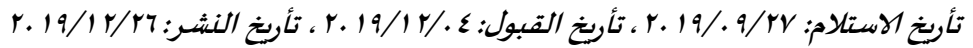

\section{الخلاصة}

اعدت دراسة مراجعية لعائلة زنابير الخصر Sphecidae في العراق، اذ اجري مسح

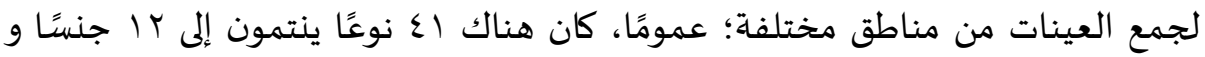

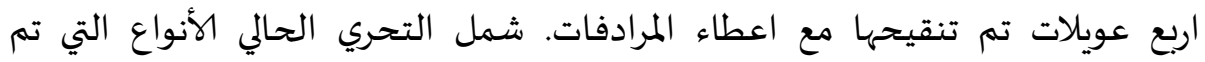

تسجيلها سابقًا في العراق، والتي لم يتم جمعها خلال التعاء المرادفات التحريات الحالية.

قدمت الدراسة التوزيع الجغرافي للأنواع، المرادفات، وغيرها من المعلومات المتعلة بها. 